\title{
LXXXI. On the kinetic theory of solids (metals) and the partition of thermal energy.-Part I
}

\section{B.M. Sen}

To cite this article: B.M. Sen (1922) LXXXI. On the kinetic theory of solids (metals) and the partition of thermal energy.-Part I, Philosophical Magazine Series 6, 43:256, 672-682, DOI: 10.1080/14786442208633928

To link to this article: http://dx.doi.org/10.1080/14786442208633928

册 Published online: 08 Apr 2009.

Submit your article to this journal $\lceil\pi$

Q View related articles $\asymp$ 
LXXXI. On the Kinetic Theory of Solids (Metals) and the Partition of Thermal Energy.-Part I. By B. M. Sen, Dacca College, Datea, Bengal*.

\section{Preface.}

r VHE Kinetic Theory of Gases has been developed in 1 great detail, but hardly any progress has been made with that of the solids. In the absence of any such welldeveloped theory, attempts are sometimes made to apply the results of the Kinetic "Theory of Gases to the case of solids, as, for example, in the theory of electric conduction. The results can hardly be regarded as satisfactory. One fundamental assumption of the Gas-theory is that the volume of the molecules is negligible in comparison with that of the gas itself. Clearly, this does not hold good even approximately for solids. Lately Nernst and others have approiched the subject from the standpoint of Planck's Quantum Theory. In the present paper an attempt has been made to develop the theory for metals on the basis of classical mechanics.

It is known that for $1^{\circ}$ rise of temperature, every atom of any metal absorbs $4 \times 10^{-16} \mathrm{erg}$ in round numbers. On the principle of equi-partition of energy, every atom having three degrees of freedom of translation, this is explained by the supposition that the translational motion absorbs one-half of this quantity of energy

$$
\left[\frac{1}{2} m v^{2}=\alpha \theta, \text { where } \alpha=2.02 \times 10^{-16}\right],
$$

and the other half is absorbed by the increase of potential energy of vibration, whose mean value is the same as that of the kinetic energy. But there are difficulties in the way, as the specitic heat increases gradually, whereas the degrees of freedom can increase only by leaps $t$. It may be pointed out that Born $\ddagger$ and others have developed the theory of crystal lattices, which gires the twenty-one constants required by the mathematical theory of elasticity. Between two neighbouring molecules there must be a force of attraction and another of repulsion which keep the molecules at their proper distance. Born assumed the form $a / p+b / p^{n}$ for the mutual potential energy, the first term representing the force of attraction and the second repulsion. But this does

* Communicated by the Author.

$\dagger$ MacLewis, 'Physical Chemistry,' vol. ii. p. 29.

† Born, Dynamik der Kristalgitter, Aufbau der Materie, where full references are given. 
not easily explain the heat expansion of the body. If heat be a form of motion, it is clear that the motion of the atoms must have something to do with the force of repulsion. I have, therefore, assumed that the repulsion is of the nature of an impulse due to what are called impacts in the Kinetic Theory of Gases. The problem thus becomes a dynamical one and the mathematies much more difficult.

Again, in order to allow impacts, it is necessary to assume that the mutual distancer of molecules are not large enough for free vibration. For simplicity I have assumed that the amplitude is small in comparison with the atomic dimensions. The results obtained completely bear out with the assumption.

It may be pointed out that the phraseology used is that of the Kinetic Theory of Gases. Use has, therefore, been made of terms such as impacts, radius of atoms, which in the light of modern researches on the structure of matter will have to be accepted in a generalized sense. Laby,

The numerical values have been taken from Kaye and

1. Some numerical values.

Let $\mathrm{N}$ be the number of molecules in a metal supposed monatomic per c.cm., and $l$ the average distance between the centres of two adjacent ones. Then $\mathrm{N} l^{3}=1^{*}$.

The number of molecules of gas per c.cm. at N.T.P.

$$
=2.75 \times 10^{19} \text {. }
$$

Let $W$ be the atomic weight, and $D$ the density of the metal. Let $w$ be the weight of one molecule of the standard gas $(0=16)$.

Then

$\mathrm{D}=$ wt. of $1 \mathrm{c.cm}$. of the metal $=\mathrm{N} . \mathrm{W} \cdot \frac{1}{2} w$.

wt. of $1 \mathrm{c.cm}$. of oxygen $=2 \cdot 75 \times 10^{19} \times 16 w=1 \cdot 429 \times 10^{-3}$.

$$
\therefore \frac{\mathrm{NW}}{\mathrm{D}}=6.16 \times 10^{23}
$$

$=$ the number of atoms in one gram atom of the metal $=\epsilon$ say.

* Strictly speaking this relation holds only for the cubical arrangement of the molecules. But for the fare-centred cubical arrangement the equation ought to be $\mathrm{N} l^{3}=\sqrt{ } 2$. This would increase the valum of $l$ given in the table by about 11 per cent. But there will be no occasion to use the arithmetical values of $l$. All that we need trouble about is the order of magnitude of $l$.

Phil. Mag. S. 6. Vol. 43. No. 256. April 1922, 2 X 
674 Prof. B. M. Sen on the Kinetic Theory of Solids

TABLE $\mathrm{I}$.

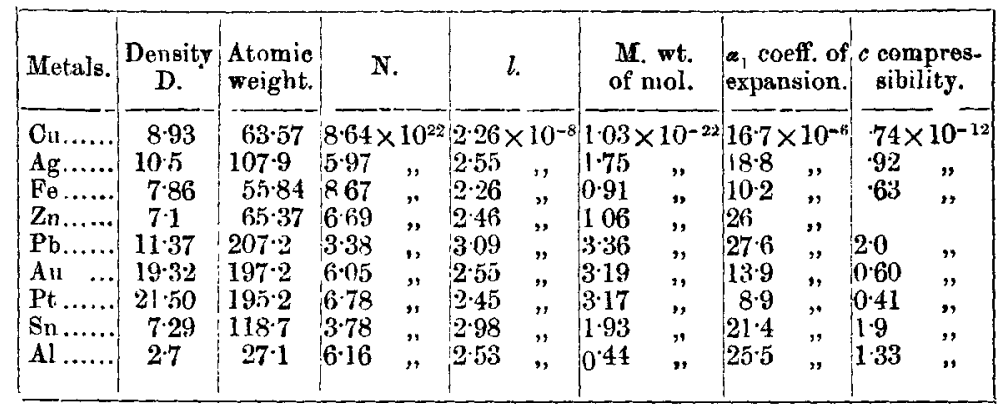

2. Arrangement of molecules in an isotropic body.

To find the number of molecules which can be arranged abrut a central one at a distance $l$ from it and from one another. The problem is the same as the determination of the number of tetrahedrons having all their edges equal to $l$ and having one vertex at the centre which can be fitted inside a sphere of radius $l$. For every tetrahedron we have an equilateral triangle of side $l$ baving its vertices on the sphere. If $a, b, c$ be the sides of the spherical triangle so formed,

$$
\begin{aligned}
a & =b=c=60^{\circ} . \\
\therefore \operatorname{Cos} \mathrm{A} & =\frac{1}{3}, \text { and } \mathrm{A}=70^{\circ} .
\end{aligned}
$$

$\therefore$ the spherical excess $=30^{\circ}$.

The total number of tetrahedrons $=\frac{4 \pi}{\pi / 6}=24$.

For each tetrahedron there are three molecules and each molecule comes in $\frac{360}{70}$, or about five times. The number of molecules, therefore, is about 14 .

\section{Rough working model.}

It is not geometrically possible to have an arrangement of molecules on a sphere so that the spherical arc between any two adjacent ones is $60^{\circ}$. $\quad B$ it in a rough model form these 14 may be supposed to be arranged as follows :-The centres all lie on a sphere of radius $l$. Two may be placed at the two poles which may he arbitrarily chosen. Three meridian circles are drawn making an angle of $60^{\circ}$ with 
each other. On each of these, beside the two at the poles, are placed four others, one at each extremity of a pair of diameters making an angle of $60^{\circ}$ with the axis. There are thus six molecules on each of these meridian circles, one at each vertex of a regular hexagon inscribed in the circle. This arrangement gives the 14 molecules arranged about the central one. It is symmetrical through the centre and has an axis.

4. The Potential Energy of displacement.

Let us consider the motion of a molecule $O$ in the direction $A B$ which we take as the direction of the axis of the model (fig. 1). Let two molecules be placed at $A$ and $B$ and let

Fig. 1.

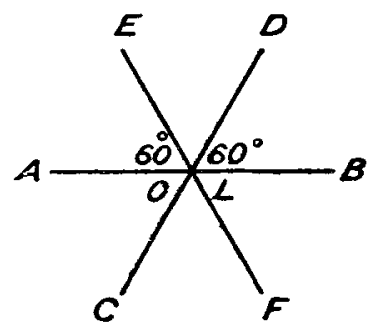

C, D, E, F be the four other molecules in the same meridian plane. so that $\mathrm{OA}=\mathrm{OB}=() \mathrm{C}=\mathrm{AE} \ldots=l$. For simplicity, we shall assume that the central one is the only one vibrating, the others being stationary in their mean positions. Let $\mathbf{L}$ be the extreme position of $\mathrm{O}$. Take $\mathrm{OL}=a$, and $r=$ the radius of the molecule. Then

$$
l=a+2 r .
$$

Let $f(l)$ be the potential energy of two molecules at a distance $l$. Then for a displacement $x$ taken small, along the axis of the molel, the potential energy due to $A$ and $B$ is

$$
f(l+x)+f(l-x)=2 f(l)+x^{2} f^{\prime \prime}(l) .
$$

The potential energy due to a pair like $C, D$

$$
=f\left(r_{1}\right)+f\left(r_{2}\right) \text { where } r_{1}=\mathrm{CL} \text {, and } r_{2}=\mathrm{DL} \text {. }
$$

Now

$$
\begin{array}{rlrl}
r_{1}^{2} & =l^{2}+x^{2}+x l . \\
\therefore \quad & r_{1} & =l+\frac{1}{2} x+\frac{3}{8} \cdot \frac{x^{2}}{l},
\end{array}
$$

neglecting higher powers of $x$, 
and

$$
\begin{gathered}
r_{2}=l-\frac{1}{2} x+\frac{3}{8} \cdot \frac{x^{2}}{l} . \\
\therefore f\left(r_{1}\right)=f(l)+\left(\frac{1}{2} x+\frac{3}{8} \frac{x^{2}}{l}\right) f^{\prime}(l)+\frac{1}{8} x^{2} f^{\prime \prime}(l), \\
\text { and } f\left(r_{2}\right)=f(l)+\left(-\frac{1}{2} x+\frac{3}{8} \frac{x^{2}}{l}\right) f^{\prime}(l)+\frac{1}{8} x^{2} f^{\prime \prime}(l) . \\
\therefore f\left(r_{1}\right)+f\left(r_{2}\right)=2 f(l)+\frac{3}{4} \frac{x^{2}}{l} f^{\prime}(l)+\frac{1}{4} x^{2} \cdot f^{\prime \prime}(l) .
\end{gathered}
$$

There are six pairs of such molecules. The total potential energy

$$
=14 f(l)+\frac{1}{2} x^{2}\left\{\frac{9 f^{\prime}(l)}{l}+5 f^{\prime \prime}(l)\right\} .
$$

It may be noted that the attractive force is $f^{\prime}(l)$. And if, as is only natural to assume, the force diminishes with the distance, $f^{\prime \prime}(l)$ is negative. And if the force varies as any power of the distance, the two terms of the variable part of the potential energy are of the same order of magnitude.

It is obvious, moreover, from general considerations that the potential energy for any displacement $x$ must be of the type $\mathrm{A}+\mathrm{B} x^{2}+\ldots$. If the coefficient of $x$ be not zero, there will be a permanent force independent of the displacement.

The equation of energy, therefore, is

$$
\mathrm{MV}^{2}+x^{2}\left\{\frac{9 f^{\prime}(l)}{l}+5 f^{\prime \prime}(l)\right\}=\text { const. . . }
$$

The motion, therefore, is simple harmonic if the coefficient of $x^{2}$ be positive. If the molecule can complete its oscillation, the average potential energy would be equal to the average kinetic energy. We assume that this is not the case, so that the molecule comes into collision with its neighbour as soon as it has described a small fraction of its path ${ }^{*}$.

\section{Order of magnitude of the Kinetic and Potential Energy terms.}

In the equation (1) above, the first term

$$
\mathrm{MV}^{2}=2 \alpha \theta
$$

is of order $10^{-14}$, for $\alpha=2 \cdot 02 \times 10^{-16}$ and $\theta$ is the temperature. From known values of the specific beat and the cuefficient of linear expansion, it will be prover that $f^{\prime}(l)$ is of order $10^{-5}$ and the maximum displacement of order $10^{-0}$. Now, $l$ being of order $10^{-8}$, the putential energy term is of order

* The reason for this assumption bas been given in the preface. 
$10^{-17}$. The error in neglecting it is therefore small. Its effect is felt at the boundary where it gives rise to a powerful surface pressure.

We neglect, therefore, the change in the potential energy and take the velocity as constant throughout the motion. Also we neglect the duration of the impact in comparison with the period of vibration. If $t_{1}$ be the time from one extreme position to the other, and $t_{2}$ the duration of the impact, $t_{2}$ will be neglected in comparison with $t_{1} *$.

\section{Heat Relations.}

To consider the heat relations of a body it is necessary to formulate a relation between the molecular kinetic energy and the temperature of the body. It may be pointed out that the equation

$$
\frac{1}{2} M V^{2}=\alpha \theta
$$

for gases does not follow from dynanical considerations, but is a direct consequence of Boyle and Charles' law. As a matter of fact, we may lake it as the definition of temperature. For a solid we take

$Q=$ the average external kinetic energy of a molecule $=\frac{1}{2} \mathrm{MV}^{2}$.

$\mathrm{P}=$ the internal kinetic energy per molecule, $i$. e. the energy of motion relative to the centre. These are, of course, additive in the ordinary way.

If $Q$ be an analytis function of $\theta$ vanishing with it, we may take

$$
\mathrm{Q}=\alpha \theta+\alpha_{1} \theta^{2}+\ldots
$$

We shall follow the usage of the Gas-theory and suppose that the temperature is determined by the average external kinetic energy of the molecule, taking

$$
\mathrm{Q}=\alpha \theta \text {. }
$$

7. Partition of Energy.

When the temperature of a solid is raised by $1^{\circ} \mathrm{C}$., the quantity of energy absorbed per unit volume is $D s \mathrm{~J}, s$ being the specific heat and $J=4 \cdot 18 \times 10^{7}$ ergs. Now Dulong and Petit's law states that $W s$ is approximately constant for all elements. Energy imparted to every molecule

$$
=\frac{\mathrm{D} s \mathrm{~J}}{\mathrm{~N}}=\frac{\mathrm{Ws}}{\epsilon} . \mathrm{J} \text {. }
$$

* Cf. J. J. Thomson, 'Corpuscular Theory of Matter,' p. 93. 
678 Prof. B. M. Sen on the Kinetic Theory of Solids

For a gas such as oxygen,

$$
\begin{gathered}
c_{p}=\cdot 24, \quad \gamma=1 \cdot 4 ; \\
\therefore \quad c_{v}=\frac{\cdot 24}{1 \cdot 4} .
\end{gathered}
$$

Energy absorbed by each molecule for $1^{\circ}$ rise of temperature

$$
=\frac{\mathrm{D}}{\mathrm{N}} c_{v} \mathrm{~J}=3 \cdot 74 \times 10^{-16},
$$

whereas the external kinetic energy is increased by

$$
\alpha=2 \cdot 02 \times 10^{-16} \text {. }
$$

In the Kinetic Theory of Gases this difference is explained by the diatomic nature of the molecules, there being no intermolecular forces*. A portion may also be accounted for by the absorption by the electrons. But for solids the matter is complicated by the presence of intermolecular forces. against which work has to be done by the heat expansion.

For a solid we assume that the total energy imparted is split up into three portions :-

(1) a quantity a absorbed in increasing the external kinetic energy $\mathrm{Q}=\frac{1}{2} \mathrm{MV}^{2}=\alpha \theta$, where $\alpha=2.02 \times 10^{-16}$, and $\theta$ the temperature.

(2) a quantity $\mathrm{B}$ absorbed in increasing the kinetic energy of rotation relative to tho centre. This is presumably absorbed by the electrons in the outer rings. We take

$$
\mathrm{P}=\mathrm{A}+\mathrm{B} \theta=\frac{1}{2} n \cdot m v^{2}
$$

where $A$ represents the store of energy, $n$ the number of electrons taking part in the absorption, $m$ the mass of an electron for velocities small in comparison with that of light, and $v$ the velocity.

(3) a quantity $\beta$ absorbed in increasing the potential energy of the molecules, $i$. e. in doing work against the inter-molecular forces.

$$
\therefore \quad \alpha+\beta+\mathrm{B}=\frac{\mathrm{W} s}{\epsilon} \mathrm{J} .
$$

Taking $\frac{1}{2} \mathrm{MV}^{2}=\alpha \theta$ for $\mathrm{Cu}$ at $\theta=17^{\circ} \mathrm{C}$.,

$$
\mathrm{V}=3.7 \times 10^{4} \text {. }
$$

Thus for metals at ordinary temperatures $\mathrm{V}$ is of order $10^{4}$. A table of values is given for solids and gases. It may

* Jeans, 'Dynamical Theory of Gases,' art. 270. 
be noted that at first sight the similarity in the values of the total gain of energy for solids and gases may tempt one to apply the diatomic explanation to solids. But the value of $\mathrm{N}$ has been calculated on the monatomic basis. If the molecules be taken as diatımic the numbers in the third column will be doubled and the similarity will disappear.

Total gain of energy per molecule for $1^{\circ} \mathrm{C}$.

$$
=s \mathrm{~W} \times 6.8 \times 10^{-17} \text {. }
$$

TABLE II.

\begin{tabular}{|c|c|c|c|c|c|c|}
\hline Metals. & $\begin{array}{l}\text { Specific } \\
\text { beat, }\end{array}$ & $\begin{array}{l}\text { Atomic } \\
\text { weight. }\end{array}$ & $\begin{array}{l}\text { Total gain } \\
\text { of energy } \\
\text { per mol. }\end{array}$ & $\beta+\mathrm{B}$. & $\beta$ (art. 9). & F (art. 9). \\
\hline $\begin{array}{l}\mathrm{Cu} \ldots \ldots . \\
\mathrm{Ag} \ldots \ldots . \\
\mathrm{Au} \ldots . . . \\
\mathrm{Pt} \ldots \ldots . \\
\mathrm{Fe} \ldots \ldots \\
\mathrm{Pb} \ldots \ldots . \\
\mathrm{Sn} \ldots \ldots . \\
\mathrm{Zn} \ldots \ldots\end{array}$ & $\begin{array}{l}\cdot 09 \\
.053 \\
\cdot 03 \\
.031 \\
\cdot 1 \\
\cdot 03 \\
\cdot 05 \\
.09\end{array}$ & $\begin{array}{r}63 \\
108 \\
197 \\
195 \\
56 \\
207 \\
119 \\
65\end{array}$ & 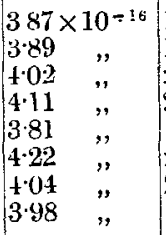 & 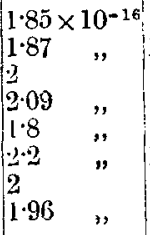 & 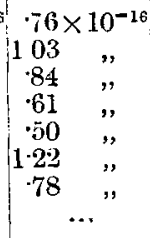 & 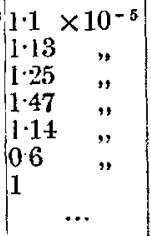 \\
\hline Gase: & $\mathrm{C}_{v}$ & Density. & & & & \\
\hline $\begin{array}{lll}A & \ldots . . \\
\mathrm{H} & \ldots \ldots . \\
\mathrm{N} & \ldots \ldots . \\
\mathrm{O} & \ldots \ldots . \\
\mathrm{CO} & \ldots . \\
\mathrm{CO}_{2} & \ldots\end{array}$ & $\begin{array}{l}\cdot 0746 \\
2 \cdot 40 \\
\cdot 175 \\
\cdot 17 \\
\cdot 17 \\
\cdot 165\end{array}$ & $\begin{array}{l}0.09 \times 10^{-3} \\
1.25 \quad " \\
1.43 \quad " \\
1.25 \% \\
1.976 ",\end{array}$ & 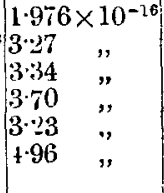 & & & \\
\hline
\end{tabular}

8. The Amplitude of Molecular Vibration a.

We revert to the consideration of a vibrating molecule $O$ surrounded by fourteen others. stationary in their mean positions. Its average velocity in any direction may be taken to be $V / \sqrt{ } 3$. At each collision this forward veloeity is reversed. Thus there is a change of momentum at the point $2 M V / \sqrt{ } 3$ at each collision, or $\frac{M V}{\sqrt{3}\left(t_{1}+t_{2}\right)}$ per sec., $2\left(t_{1}+t_{2}\right)$ being the period of vibration. Neglecting $t_{2}$ the duration of the collision, and putting $t_{1}=2 \sqrt{ } 3 a / V$, the change of momentum becomes $\frac{\mathrm{MV}^{2}}{6 a}=\frac{\alpha \theta}{3 a}$. There being $\mathrm{N}^{2 / 3}$ molecules per sq. cm., the pressure at the surface within the mass of the metal is $\mathrm{N}^{2 / 3} \cdot \frac{\alpha \theta}{3 a}$ per sq. cm. 
We now calculate the pressure at the surface due to the molecular forces, the atmospheric pressure being of course negligible. We take into account only seven of the fourteen molecules lying on one side of the equatorial plane. The potential energy in the displaced pusition, $x$ being the displacement from the mean position,

$$
\begin{aligned}
& =f(l-x)+6 f\left(l-\frac{1}{2} x\right) \\
& =7 f(l)-4 x f^{\prime}(l) .
\end{aligned}
$$

Force along the normal

$$
=4 f^{\prime}(l)=4 \mathrm{~F},
$$

where $\mathrm{F}$ is the force between two molecules.

Pressure at the surface $=4 \mathrm{~N}^{2 / 3} . \mathrm{F}$.

$$
\begin{aligned}
\therefore \quad \frac{\alpha \theta}{3 \alpha} & =4 \mathrm{~F} \\
\text { or } \quad a & =\frac{\alpha \theta}{12 \mathrm{~F}^{\mathrm{N}}} . . .
\end{aligned}
$$

9. Compressibility.

We proceed to find the simplest of the elastic constants, viz. compressibility, on the basis of our hypothesis. The work done against the molecular forces $=\beta$ per molecule for $1^{\circ}$ rise of temperature.

Now the pressure at the surface $=4 \mathrm{~F} . \mathrm{N}^{2 / 3}$.

Work done against the surface pressure $=\mathrm{N}^{2 / 3} .12 \mathrm{~F} \alpha_{1}$, where $\alpha_{1}$ is the coefficient of linear expansion.

Work done against the inter-molecular forces per unit volume $=i \mathrm{~F} \delta l . \mathrm{N}=7 \mathrm{~F} \alpha_{1} \mathrm{~N}^{2 / 3}$, there being fourteen molecules arranged about each individual molecule and $\delta a=\delta l$.

$$
\begin{aligned}
& \therefore \quad \mathrm{N} \beta=19 \mathrm{~F} \alpha_{1} \mathrm{~N}^{2 / 3} . \therefore \quad \mathrm{F}=\frac{\beta}{19 \alpha_{1} l} . . . \\
& \therefore \quad a=\frac{\alpha \theta}{12 \mathrm{~F}}=\frac{19 \alpha \theta \cdot \alpha_{1} l}{12 \beta} .
\end{aligned}
$$

Now the surface pressure $=4 \mathrm{~F} \cdot \mathrm{N}^{2 / 3}=\frac{4 \mathrm{~N} \beta}{19 \alpha_{1}}$.

If the pressure be increased by one dyne without change of temperature, it increases by $\frac{19 \alpha_{1}}{4 N \beta}$ th part of itself.

$$
\begin{aligned}
& \therefore \quad \frac{\delta a}{a}=-\frac{19 \alpha_{1}}{4 \mathrm{~N} \beta} . \\
& \therefore \quad \frac{\delta l}{l}=-\frac{a}{l} \cdot \frac{19 \alpha_{1}}{4 \mathrm{~N} \beta} .
\end{aligned}
$$


and the Partition of Thermal Energy.

$$
\begin{array}{r}
\text { Compressibility } \mathrm{C}=\frac{57 \alpha_{1} a}{4 \mathrm{~N} \beta l}=\frac{57 \alpha_{1}}{4 \mathrm{~N} \beta l} \times \frac{19 a \theta}{12 \beta} a_{1} l . \\
\therefore \quad \beta^{2}=\frac{19 \times 19 \times \alpha_{1}^{2}}{16 \mathrm{C} N} \alpha \theta \text { or } \beta=\frac{19}{4} \alpha_{1} \sqrt{\frac{\alpha}{\mathrm{CN}}} .
\end{array}
$$

Taking $\quad \mathrm{C}=\cdot 74 \times 10^{-12}$ and $\theta=17^{\circ} \mathrm{C}$., we get $\quad \beta=76 \times 10^{-16}$ for $\mathrm{Cu}$.

Substituting this value of $\beta$, we have for Cu

$$
\mathrm{F}=1 \cdot 1 \times 10^{-5} \text { and } a=4 \cdot 4 \times 10^{-10} \text {. }
$$

The value of $\beta$ tor the different metals has been calculated and shown in the second table. The values of $B$ are obtained by difference.

10. The calculation of Young's modulus gives good support to the previous work. Suppose that the body is stretched so that $l$ increases by $x$.

$$
\therefore \delta l=\delta a=r \text {, where } l=a+2 r .
$$

Putting $a+x$ instead of $a$ in art. 8, the half-period of vibration

$$
t_{1}=2 \sqrt{ } 3(a+x) / \mathrm{V} \text {. }
$$

The change of momentum per sec. is, therefore,

$$
\frac{M V^{2}}{b(a+x)}=\frac{M V^{2}}{6 a}\left(1-\frac{x}{a}\right)
$$

The force of repulsion due to impacts is diminished by $\frac{\mathrm{MV}^{2} x}{6 a^{2}}$.

The force of attraction

$$
=f^{\prime}(l+x)-f^{\prime}(l)+x f^{\prime \prime}(l)
$$

$f^{\prime \prime}(l)$ being of course negative.

The total change in the force of attraction per molecule

$$
=w\left\{\frac{M V^{2}}{6 a^{2}}+f^{\prime \prime}(l)\right\},
$$

and the total change per unit area

$$
=x \mathrm{~N}^{2 / 3} \cdot\left\{\frac{M V^{2}}{6 a^{2}}+f^{\prime \prime}(l)\right\} .
$$

Now the extension is $x / l$. Young's modulus is, therefore,

$$
\mathrm{N}^{2 / 3} \cdot l\left\{\frac{\mathrm{JV} \mathrm{V}^{2}}{6 a^{2}}+f^{\prime \prime}(l)\right\}=\frac{1}{l}\left\{\frac{\mathrm{MV}^{2}}{6 a^{2}}+f^{\prime \prime}(l)\right\} .
$$


It is known that the value of Young's modulus for the common metals is of the order $10^{12}$. Taking the case of Cu by way of illustration, the tirst term $=5 \times 10^{12}$ roughly.

The experimental value $=1.23 \times 10^{12}$.

$\therefore \frac{1}{l} f^{\prime \prime}(l)$ is of order $10^{12}$, which makes $f^{\prime \prime}(l)$ of order $10^{4}$.

The potential energy term in the equation of energy in art. 4 is therefore of order $10^{-16}$.

\section{Residual Rays.}

We can make a rough calculation of the frequency of vibration of the molecules at the surface. We know that for a Ca molecule,

$$
\begin{aligned}
& \mathrm{V} \text { is of order } 10^{4}, \\
& \mathrm{M} ", \quad, \quad 10^{-22}, \\
& \mathrm{~F} ", \quad 10^{-5} .
\end{aligned}
$$

Neglecting the variations of the force, the frequency $\nu=\frac{\mathrm{F}}{2 \mathrm{MV}}$, which is of order $10^{12}$.

This agrees with the experimental values*.

\section{Conclusion.}

We have, therefore, arrived at a consistent theory of the solid state. Starting from known values of the specific heat, compressibility, and the coefficient of linear expansion, we have investigated the amplitude of molecular vibration and the inter-molecular forces. The partition of the gain of thermal energy has been dealt with. We have found. that for $\mathrm{Cu}$, for exumple,

(1) $2.02 \times 10^{-10} \mathrm{erg}$ is absorbed by each molecule to increase the energy of translational motion,

(2) $1.2 \times 10^{-16}$ erg is absorbed by the rotational motion, and presumably increases the energy of the electrons,

(3) $\cdot 76 \times 10^{-16} \mathrm{erg}$ is absorbed in doing work against the inter-molecular forces.

The cases of cubical and face-centred cubical crystals have been dealt with in the next paper; the results obtained do not differ materially from those obtained here.

* McLewis, 'Physical Chemistry,' vol, ii. p. 58. 